\title{
REMARKS ON SEMISEPARATION OF LATTICES
}

\author{
CARMEN D. VLAD \\ Department of Mathematics \\ Pace University \\ New York, N.Y.10038,U.S.A. \\ (Received April 10, 1990 and in revised form November 1, 1990)
}

ABSTRACT. This paper is concerned primarily with conditions for semiseparation and separation of lattices. These conditions are expressed in terms of the general Wallman space.

KEY WORDS AND PHRASES. Lattice, measure, filter.

1980 AMS SUBJECT CLASSIFICATION CODE. $28 \mathrm{C} 15$.

1. INTRODUCTION.

Let $X$ be an abstract set and $\mathscr{L}_{1}$ and $\mathscr{L}_{2}$ lattices of subsets of $X$ such that $\mathscr{L}_{1} \subset \mathscr{L}_{2}$ If $A \cap B=\emptyset, A \in \mathcal{L}, B \in \mathscr{L}_{2}$ implies there exists a $C \in \mathcal{L}$, such that $C \supset B$, and $A \cap C=\emptyset$ then $\mathscr{L}_{1}$ is said to semiseparate $\mathscr{L}_{2}$. This notion is important in topological spaces, where $\mathscr{L}_{1}$ and $\mathscr{L}_{2}$ are specific lattices such as, for example, the zero-sets and the closed sets.

We investigate this property in terms of associated measures and outer measures associated with the respective lattices, and also with respective Wallman spaces. This gives us new conditions for one lattice to semiseparate another, and gives additional facts pertaining to the measures. These investigations are carried out in sections 3 and 4 . In section 2 we give some background material, which is fairly standard by now, and can be found in [1-3]. This material has been added mainly for the reader's convenience.

2. BACKGROUND AND NOTATIONS.

Let $X$ be an abstract set and $\mathscr{L}$ a lattice of subsets of $X$. It is assumed that $\emptyset, x \in \mathscr{L}$. We denote by $a(\mathcal{L})$ the algebra generated by $\mathcal{L} ; \delta(\mathscr{L})$, the lattice of all countable intersections of sets from $\mathcal{L}$.

DEFINITION $2.1 \mathscr{L}$ is:

delta lattice ( $\delta$-lattice) if $\mathcal{L}$ is closed under countable intersections.

complement generated if $L \in \mathscr{L}$ implies $L=\bigcap_{n=1}^{\infty} L_{n}^{\prime}, L_{n} \in \mathscr{L}$ (where prime denotes complement).

disjunctive if for $x \in X$ and $L_{1} \in \mathcal{L}$ such that $x \notin L_{1}$ there exists $L_{2} \in \mathcal{L}$ with $x \in L_{2}$ and $\mathrm{L}_{1} \cap \mathrm{L}_{2}=\emptyset$.

normal if for any $L_{1}, L_{2} \in \mathscr{L}$ with $L_{1} \cap L_{2}=\emptyset$, there exist $L_{3}, L_{4} \in \mathscr{L}$ with $L_{1} \subset L_{3}^{\prime}$, $L_{2}<L_{4}^{\prime}$ and $L_{3}^{\prime} \cap L_{4}^{\prime}=\emptyset$.

compact if for any collection $\left\{L_{\alpha}\right\}$ of sets of $\mathscr{L}$ with $\cap L_{\alpha}=\emptyset$, there exists a finite subcollection with empty intersection. 
countably compact if for any countable collection $\left\{L_{\alpha}\right\}$ of sets of $\mathscr{L}$ with $\cap L_{\alpha}=\emptyset$, there exists a finite subcollection with empty intersection.

Lindeluf if for any collection $\left\{L_{\alpha}\right\}$ of sets of $\mathscr{L}$ with $\cap L_{\alpha}=\emptyset$, there exists a countable subcollection with empty intersection.

$T_{2}$-lattice if for $x, y \in X, x \neq y$, there exist $L_{1}, L_{2} \in \mathcal{L}$ such that $x \in L_{1}^{\prime}, y \in L_{2}^{\prime}$ and $L_{1} \cap L_{2}^{\prime}=\emptyset$.

DEFINITION 2.2 We give now some measure terminology which will be used throughout. $M(\mathcal{L})$ denotes the set of finite valued bounded finitely additive non-trivial measures on $a(\mathscr{L})$. Without loss of generality may assume throughout that all mea sures are non-negative. A measure $\mu \in M(\mathscr{L})$ is called:

$\sigma$-smooth on $\mathcal{L}$ if for all sequences $\left\{L_{n}\right\}$ of sets of $\mathcal{L}$ with $L_{n} \downarrow \theta, \mu\left(L_{n}\right) \rightarrow 0$. $\sigma$-smooth on $Q(\mathcal{L})$ if for all sequences $\left\{A_{n}\right\}$ of sets of $Q(\mathcal{L})$ with $A_{n} \downarrow \emptyset$, $\mu\left(A_{n}\right) \longrightarrow 0$ (i.e. countably additive measures on $a(\mathscr{L})$ ).

$\mathscr{L}$-regular if for any $A \in Q(\dot{L}), \mu(A)=\sup \{\mu(L) / L C A, L \in \mathscr{L}\}$.

In addition we denote by $M_{R}(\mathscr{L})$, the set of $\mathscr{L}$-regular measures of $M(\mathscr{L}) ; M_{\sigma}(\mathscr{L})$ the set of $\widetilde{G}$-smooth measures on $\mathscr{L}$ of $M(\mathscr{L})$; $M^{\mathscr{\sigma}}(\mathscr{L})$, the set of $\mathscr{\sigma}$-smooth measures on $\boldsymbol{Q}(\mathscr{L})$ of $M(\mathscr{L}) ; M_{R}^{\mathscr{F}}(\mathscr{L})$, the set of $\mathscr{L}$-regular measures of $M^{\mathscr{\sigma}}(\mathscr{L})$.

$I(\mathscr{L}), I_{R}(\mathscr{L}), I_{\mathscr{S}}(\mathscr{L}), I_{R}^{\mathscr{E}}(\mathscr{L})$ are the subsets of the corresponding $M$ 's which consist of the non-trivial zero-one valued measures.

DEFINITION 2.3 For $\mu \in M(\mathscr{L})$, the support of $\mu$ is $S(\mu)=n\{L \in \mathscr{L} / \mu(L)=\mu(X)\}$. $\mathcal{L}$ is replete iff for any $\mu \in I_{R}^{\sigma}(\mathscr{L}), s(\mu) \neq \emptyset$.

DEFINITION 2.4 A filter in $\mathscr{L}$ is a subset of $\mathscr{L}, \mathscr{F}$, satisfying the condi tions: $\emptyset \notin \mathscr{F} ; \mathcal{F}$ is closed under finite intersections; if $A \in \mathcal{F}, B \in \mathcal{L}$ and $A \subset B$ then $B \in \mathscr{F}$.

An ultrafilter in $\mathcal{L}$ is a maximal filter in $\mathscr{L}$ ( relative to the partial order on the collection of filters in $\mathscr{L}$ given by inclusion).

An $\mathscr{L}$-filter $\mathcal{F}$ is prime if given $A, B \in \mathscr{L}$ such that $A \cup B \in \mathcal{F}$ then either $A \in \mathcal{F}$ or $B \in \mathscr{F}$.

There exists a one-to-one correspondence between $\mathscr{L}$-filters $\mathscr{F}$ and elements of $\pi(\mathscr{L})=\{\pi$, defined on $\mathcal{L}$, monotone and $\pi(A \cap B)=\pi(A) \pi(B), A, B \in \mathscr{L}\}$ defined by $\pi(L)=1$ iff $L \in \mathcal{F}$. There exists a one-to-one correspondence between $\mathcal{L}$-filters $\mathcal{F}$ with countable intersection property and $\mathbb{J}_{\mathscr{\sigma}}(\mathscr{L})$, where $\mathbb{J}_{\mathscr{\sigma}}(\mathscr{L})=\{\pi \in \mathscr{K}(\mathscr{L})$ such that if $T\left(L_{n}\right)=1$ all $n$ where $L_{n} \in \mathcal{L}$ then $\left.\bigcap L_{n} \neq \emptyset\right\}$. There exists a one-to-one correspondence between all elements of $I_{R}(\mathcal{L})$ and all $\mathcal{L}$-ultrafilters. There exists a one-to-one correspondence between all elements of $I_{R}^{\mathscr{F}}(\mathcal{L})$ and all $\mathcal{L}$-ultrafilters with the countable intersection property. The correspondence is given by the follo wing rule: with each $\mathscr{L}$-ultrafilter $\mathscr{\mathcal { F }}$ we associate the zero-one valued measure defined on $\boldsymbol{a}(\mathscr{L})$ by

$$
\mu(E)= \begin{cases}1 & \text { if there exists } A \in \mathscr{F}, A \subset E \\ 0 & \text { if there exists } A \in \mathscr{F}, A \subset E^{\prime} .\end{cases}
$$

There exists a one-to-one correspondence between all elements of $I(\mathscr{L})$ and all prime $\mathscr{L}$-filters, given by the following rule: with each $\mu \in I(\mathcal{L})$ we associate the prime $\mathscr{L}$-filter given by $\mathscr{F}=\{A \in \mathscr{L} / \mu(A)=1\}$. This correspondence induces a one-to-one correspondence between prime $\mathscr{L}$-filters with the countable intersection property and $I_{G}(\mathscr{L})$.

REMARK. It is not difficult to see in light of the above correspondences that $\mathscr{L}$ is normal iff for each $\mu \in I(\mathscr{L})$, there exists a unique $\nu \in I_{R}(\mathscr{L})$ such that $\mu \leq \nu(\mathscr{L})$ (i.e. $\mu(L) \leqslant \nu(L)$ for all $L \in \mathscr{L}$ ). 
3. SEMISEPARATION.

DEFINITION 3.1 Let $\mathcal{L}$ be a lattice of subsets of $X$, let $\mu \in I(\mathcal{L})$ and $E \subset X$ and define $\mu^{\prime}(E)=\inf \left\{\mu\left(L^{\prime}\right) / E \subset L^{\prime}, L \in \mathscr{L}\right\}$.

THEOREM 3.1 Let $\mathscr{L}$ be a lattice of subsets of $x$ and let $\mu \in I(\mathscr{L})$. The following statements are true:

a) $\mu^{\prime}$ is finitely subadditive;

b) $\mu \in I_{R}(\mathscr{L})$ iff $\mu=\mu^{\prime}(\mathscr{L})$;

c) Let $\mu \in I_{R}(\mathscr{L})$ and $\rho \in I_{R}\left(\mathscr{L}^{\prime}\right)$ such that $\mu \leqslant \rho(\mathscr{L})$. $\mathscr{L}$ is normal iff $\mu^{\prime}=\rho^{\prime}(\mathscr{L})$ for all such $\mu$ and $\rho$.

PROOF. a) Since $\mu \in I(\mathscr{L})$ it is clear that $\mu^{\prime}(E)=\inf \left\{\sum_{i=1}^{n} \mu\left(L_{i}^{\prime}\right) / E \subset \bigcup_{i=1}^{n} L_{i}^{\prime}, L_{i} \in \mathcal{L}\right\}$ and therefore $\mu^{\prime}$ is finitely subadditive.

b) For $\mu \in I_{R}(\mathscr{L}), \mu(A)=\inf \left\{\mu\left(L^{\prime}\right) / A \subset L^{\prime}, L \in \mathscr{L}\right\}=\mu^{\prime}(A), \quad A \in \mathscr{L}$.

c) Suppose $\mu \leq \rho\left(\mathscr{L}^{\prime}\right)$. Then $\rho \leqslant \mu(\mathscr{L})$ and since $\mu \in I_{R}(\mathscr{L})$ it follows that $\mu=\mu^{\prime}(\mathscr{L})$. Then $\rho \leqslant \mu=\mu^{\prime} \leqslant \rho^{\prime}$ on $\mathscr{L}$. Suppose $\mathscr{L}$ is normal, let $A \in \mathscr{L}$ and suppose that $\mu(A)=0$. Since $\mu \in I_{R}(\mathscr{L})$, there exists $L \subset A^{\prime}, L \in \mathscr{L}$ with $\mu(L)=1$. But $A \cap L=\emptyset$ implies there exist $C^{\prime}, D^{\prime}$ such that $A \subset C^{\prime}, L C D^{\prime}, C^{\prime} \cap D^{\prime}=\varnothing, C, D \in \mathscr{L}$. Then we have $A \subset C^{\prime} C$ $C D C L^{\prime}$ and $\rho\left(C^{\prime}\right) \leqslant \rho(D) \leqslant \mu(D) \leqslant \mu\left(L^{\prime}\right)=0$. So, $\rho\left(C^{\prime}\right)=0$, i.e. $\rho^{\prime}(A)=0$. A was arbitrary in $\mathscr{L}$, then $\mu^{\prime}=\rho^{\prime}$ on $\mathscr{L}$. Conversely, suppose that $\mu^{\prime}=\rho^{\prime}(\mathscr{L})$ with $\mu$ and $\rho$ as before. Let $\mu \in I(\mathscr{L}), \mu_{1}, \mu_{2} \in I_{R}(\mathscr{L})$ with $\mu \leq \mu_{1}(\mathscr{L}), \mu \leq \mu_{2}(\mathscr{L})$. But $\mu \leqslant \rho \in I_{R}\left(\mathscr{L}^{\prime}\right)$ on $\mathscr{L}^{\prime}$, so we have $\rho \leqslant \mu \leqslant \mu$, on $\mathscr{L}$ and $\rho \leqslant \mu \leqslant \mu_{2}$ on $\mathscr{L}$. By the assumption, $\mu_{1}^{\prime}=\rho^{\prime}$ and $\mu_{2}^{\prime}=\rho^{\prime}$ on $\mathscr{L}$ and therefore $\mu_{1}=\mu_{1}^{\prime}=\rho^{\prime}=\mu_{2}^{\prime}=\kappa_{2}$ i.e. $\mathscr{L}_{\text {normal }}$.

DEFINITION 3.2 Let $\mathscr{L}$ be a lattice of subsets of $X$. The Wallman topology is obtained by taking all $\mathrm{W}(\mathrm{L})=\left\{\mu \in \mathrm{I}_{\mathrm{R}}(\mathscr{L}) / \mu(L)=1\right\}, L \in \mathscr{L}$ as a base for the closed sets in $I_{R}(\mathcal{L}) . I_{R}(\mathcal{L})$ with the Wallman topology is called the general Wallman space associated with $X$ and $\mathscr{L}$. We assume that $\mathcal{L}$ is disjunctive. Then if $A \in Q(\mathscr{L})$, let $W(A)=\left\{\mu \in I_{R}(\mathcal{L}) / \mu(A)=1\right\}$. The following statements are true:

a) $W(A \cup B)=W(A) \cup W(B)$

b) $W(A \cap B)=W(A) \cap W(B)$

c) $W\left(A^{\prime}\right)=W(A)^{\prime}$

d) $A \supset B$ iff $W(A) \supset W(B)$

e) $a(w(\mathscr{L}))=w(Q(\mathscr{L}))$.

It is known that $W(\mathscr{L})$ is disjunctive and that the topologicai space $\left(I_{R}(\mathscr{L}), \operatorname{tW}(\mathscr{L})\right)$ ) is compact and $T_{1}$ and if $\mathscr{L}$ is disjunctive it is $T_{2}$ iff $\mathscr{L}$ is normal.

THEOREM 3.2 Let $\mathscr{L}_{1} \subset \mathscr{L}_{\mathbf{2}}$ be two lattices of subsets of $x$. Suppose that $\mathscr{L}_{\mathbf{2}}$ is disjunctive and $\mathscr{L}$, is normal and consider the restriction map $\Psi: I_{R}\left(\mathscr{L}_{2}\right) \rightarrow I_{R}\left(\mathscr{L}_{)}\right)$ Then:

a) $\Psi\left(W_{2}\left(L_{2}\right)\right)=\bigcap\left\{W_{1}\left(L_{1 \alpha}\right) / L_{2} \subset L_{1 \alpha}, L_{1 \alpha} \in \mathscr{L}_{1}, L_{2} \in \mathscr{L}_{2}\right\}$ where $W_{1}\left(L_{1 \alpha}\right)$ and $W_{2}\left(L_{2}\right)$ are basic closed sets with respect to the Wallman topologies.

b) $\mathscr{L}$, semiseparates $\mathscr{L}_{\mathbf{Z}}$.

PROOF. a) Since $\left.W_{2}\left(L_{2}\right)\right)$ is closed in $W_{2}\left(\mathscr{L}_{2}\right)$, it is compact and since $\Psi$ is continuous, $\Psi\left(W_{2}\left(L_{2}\right)\right)$ is compact. $\mathscr{L}_{1}$ is normal, so $I_{R}\left(\mathscr{L}_{1}\right)$ is compact and $T_{2}$ and therefore $\Psi\left(W_{2}\left(L_{2}\right)\right)$ is closed. Then $\psi\left(W_{2}\left(L_{2}\right)\right)=\bigcap_{\alpha} w_{1}\left(L_{1 \alpha}\right)$, where $L_{1 \alpha} \in \mathscr{L}_{1}$ and since $\mathscr{L}_{2}$ is disjunctive, $L_{2} \subset L_{1 \alpha}$ for all $\alpha$.

b) Let $L_{2} \in \mathscr{L}_{2}$ and $L_{1} \in \mathscr{L}$, with $L_{2} \cap L_{1}=\emptyset$. Then $W_{2}\left(L_{2}\right) \cap W_{2}\left(L_{1}\right)=\emptyset$, which implies $\psi\left(W_{2}\left(L_{2}\right)\right) \cap W_{1}\left(L_{1}\right)=\emptyset$. For if $\mu \in W_{1}\left(L_{1}\right)$ and if $\mu=\psi(\nu)$ with $\nu \in W_{2}\left(L_{2}\right)$ then $\nu\left(L_{2}\right)=1$ and $\nu\left(L_{1}\right)=\mu\left(L_{1}\right)=1$, contradiction. Thus $\psi\left(W_{2}\left(L_{2}\right) \cap W_{1}\left(L_{1}\right)=\emptyset\right.$. By a) we have then $\left\{w_{1}\left(L_{1 \alpha}\right) / L_{2}<L_{1 \alpha}, L_{1 \alpha} \in \mathscr{L}_{1}\right\} \cap W_{1}\left(L_{1}\right)=\emptyset$. Since $W_{1}\left(\mathscr{L}_{1}\right)$ is compact it follows 
that $\left.\hat{\overbrace{}}_{i=1}^{n} W_{1}\left(L_{1 \alpha_{i}}\right) / L_{2} \subset L_{1 \alpha_{i}}, L_{1 \alpha_{i}} \in \mathscr{L},\right\} \cap W_{1}\left(L_{1}\right)=\emptyset$. Then $L_{2} \subset \bigcap_{i=1}^{n} L_{1} \alpha_{i}=A \in \mathscr{L}$, and $A \cap \mathrm{L}_{1}=\emptyset$ which proves that $\mathscr{L}_{1}$ semiseparates $\mathscr{L}_{2}$.

COROLLARY 3.1 Let $\mathscr{L}$ be a lattice of subsets of $X$. Then the following statements are equivalent:

a) $I(\mathscr{L})=I_{R}(\mathscr{L})$

b) $\mathscr{L}$ semiseparates $\mathfrak{a}(\mathscr{L})$

c) $\mathscr{L}=\mathscr{L}^{\prime}$

d) $I_{R}\left(\mathscr{L}^{\prime}\right)=I_{R}(\mathscr{L})$

PROOF. a) $\Rightarrow$ b): $\boldsymbol{a}(\mathscr{L})$ is disjunctive. $\mathscr{L}$ is normal, since for $\mu \in I(\mathscr{L})=I_{R}(\mathscr{L})$ we have $\mu \leqslant \mu(\mathscr{L})$. Consider the restriction map $\boldsymbol{\Psi}: \mathrm{I}_{\mathrm{R}}(\boldsymbol{a}(\mathcal{L}))=\mathrm{I}(\boldsymbol{a}(\mathcal{L})) \rightarrow \mathrm{I}_{\mathrm{R}}(\mathcal{L})$. $=\mathrm{I}(\mathscr{L})$. By Theorem 3.2 it follows that $\mathscr{L}$ semiseparates $\boldsymbol{Q}(\mathscr{L})$. b) $\Rightarrow \mathrm{c})$ : Let $L \in \mathscr{L}$; then $L^{\prime} \in \mathbb{Q}(\mathscr{L})$. Since $\mathscr{L}$ semiseparates $\boldsymbol{Q}(\mathscr{L})$ there exists $A \in \mathscr{L}, L^{\prime} \subset A$ and $A \cap L=\emptyset$, i.e. $A \subset L^{\prime}$. Therefore $L^{\prime} \subset A \subset L^{\prime}$ i.e. $A=L^{\prime} \in \mathcal{L}$, so $\mathscr{L}=\mathscr{L}^{\prime}$. c) $\Rightarrow$ d), clearly. d) $\Rightarrow$ a): Let $\mu \in I(\mathscr{L})$ and $\nu \in I_{R}(\mathscr{L}), \mu \leqslant \nu(\mathscr{L})$ and suppose that $\mu(A)=0, \nu(A)=1, A \in \mathscr{L}$. But $\nu \in I_{R}\left(\mathscr{L}^{\prime}\right)$, therefore there exists $L^{\prime} \subset A$, $\tilde{L} \in \mathscr{L} w^{\prime}$ th $\nu\left(\tilde{L}^{\prime}\right)=1$, or $\nu(\tilde{L})=0$. Then $\mu(\tilde{L})=0$ and since $A^{\prime} \subset \tilde{L}, \quad \mu\left(A^{\prime}\right) \leqslant \mu(\tilde{L})=0$ i.e. $\mu\left(A^{\prime}\right)=0$, contradiction. It follows that $\mu=\nu$ i.e. $I(\mathscr{L})=I_{R}(\mathscr{L})$.

COROLLARY 3.2 Let $\mathscr{L}, \subset \mathscr{L}_{\mathbf{2}}$ be two lattices of subsets of $x$, with $\mathscr{L}$, normal and $\mathscr{L}_{2}$ disjunctive. Consider $\nu \in I_{R}\left(\mathscr{L}_{2}\right)$ and its restriction $\mu \in I\left(\mathscr{L}_{1}\right)$. Then $\nu^{\prime}=\mu^{\prime}\left(\mathscr{L}_{1}\right)$ iff $\mathscr{L}$, semiseparates $\mathscr{L}_{2}$.

PROOF. Clearly, $\nu^{\prime} \leq \mu^{\prime}$, always. Let $L_{1} \in \mathscr{L}_{1}$ and suppose $\nu^{\prime}\left(L_{1}\right)=0$. Then $L_{1} C L_{2}^{\prime}$ $L_{2} \in \mathscr{L}_{2}$ and $\nu\left(L_{2}^{\prime}\right)=0$. By semiseparation there exists $\tilde{L}_{1} \in \mathscr{L}_{1}, L_{2} \subset \tilde{L}_{1}^{1}$ and $\tilde{L}_{1} \cap L_{1}=\emptyset$. Then $L_{1} \subset \widetilde{L}_{1}$ and $\tilde{L}_{1}^{\prime} \subset L_{2}^{\prime}$, so $\mu\left(\widetilde{L}_{1}^{\prime}\right)=0$ i.e. $\mu^{\prime}\left(L_{1}\right)=0$ and $\nu^{\prime}=\mu^{\prime}\left(\mathscr{L}_{1}\right)$. Conversely, suppose $\nu^{\prime}=\mu^{\prime}\left(\mathscr{L}_{1}\right)$. If $\mu\left(\mathrm{L}_{1}\right)=0$ then $\nu\left(\mathrm{L}_{1}\right)=0$ therefore $\nu^{\prime}\left(\mathrm{L}_{1}\right)=0, \mathrm{~L}_{1} \in \mathscr{L}_{1}$, since $\nu=\nu^{\prime}\left(\mathscr{L}_{2}\right)$. So, $\mu^{\prime}\left(L_{1}\right)=0$ i.e. $\mu=\mu^{\prime}\left(\mathscr{L}_{,}\right)$which by Theorem 3.1 implies $\mu \in I_{R}(\mathscr{L}$,$) .$ It follows by Theorem 3.2 that $\mathscr{L}$, semiseparates $\mathscr{L}_{\mathbf{2}}$.

DEFINITION 3.3 Let $\mathscr{L}$ be a lattice of subsets of $X$ and define

$$
\tilde{\mu}(E)=\inf \{\mu(L) / E \subset L, L \in \mathscr{L}\} \quad, E \subset X \text {. }
$$

THEOREM 3.3 Let $\mathscr{L}, \subset \mathscr{L}_{2}$ be two lattices of subsets of $X$ and let $\mu \in I_{R}\left(\mathscr{L}_{1}\right)$. Then $\tilde{\mu}=\mu^{\prime}\left(\mathscr{L}_{2}\right)$ iff $\mathscr{L}_{1}$ semiseparates $\mathscr{L}_{2}$.

PROOF. $\mu^{\prime}\left(L_{2}\right)=\inf \left\{\mu\left(L_{1}^{\prime}\right) / L_{2} \subset L_{1}^{\prime}, L_{1} \in \mathscr{Z}_{1}, L_{2} \in \mathscr{Z}_{2}\right\}$. By semiseparation, there exists $\tilde{L}_{1} \in \mathscr{L}$, with $L_{2} \subset \tilde{L}_{1} \subset L_{1}^{1}$. Therefore $\tilde{\mu}\left(L_{2}\right) \leqslant \mu^{\prime}\left(L_{2}\right)$. Now suppose $\tilde{\mu}\left(L_{2}\right)=0$. Then there exists $A \in \mathscr{L}_{1}, L_{2} \subset A$ and $\mu(A)=0$ and since $\mu \in I_{R}\left(\mathscr{L}_{1}\right)$, there exists $B \in \mathscr{L}_{1}$, $A \subset B^{\prime}$ and $\mu\left(B^{\prime}\right)=0$. Therefore $L_{2} \subset B^{\prime}$ and $\mu\left(B^{\prime}\right)=0$, hence $\mu^{\prime}\left(L_{2}\right)=0$. So $\tilde{\mu}=\mu^{\prime}\left(\mathscr{L}_{2}\right)$. Conversely, suppose that $\tilde{\mu}=\mu^{\prime}\left(\mathcal{L}_{2}\right)$ for all $\mu \in \mathrm{I}_{\mathrm{R}}\left(\mathscr{L}_{1}\right)$. If $\mathscr{L}_{1}$ does not semiseparates $\mathscr{L}_{2}$ then there exist $\mathrm{L}_{2} \in \mathscr{Z}_{2}, \mathrm{~L}_{1} \in \mathscr{L}$, such that $\mathrm{L}_{2} \cap \mathrm{L}_{1}=\emptyset$ but $\mathcal{F}=\left\{\tilde{\mathrm{L}}_{1} \cap \mathrm{L}_{1}\right.$, $\left.\tilde{L}_{1} \supset L_{2}, \tilde{L}_{1} \in \mathscr{L}\right\}$ has the finite intersection property, therefore there exists $\mu \in I_{R}(\mathscr{L}$,$) such that \mu\left(\tilde{L}_{1}\right)=1$ for all $\tilde{L}_{1} \in \mathscr{L}_{1}$ and $\tilde{L}_{1} \supset L_{2}$ and $\mu\left(L_{1}\right)=1$. Thus $\tilde{\mu}\left(L_{2}\right)=1$ but $\mu^{\prime}\left(\mathrm{L}_{2}\right)=0$, contradiction. Hence $\mathscr{L}$, semiseparates $\mathscr{L}_{2}$.

DEFINITION 3.4 Let $\mathcal{L}$ be a lattice of subsets of $X$, let $\mu \in I(\mathcal{L})$ and ECX and define $\mu$ " $(E)=\inf \left\{\sum_{i=1}^{\infty} \mu\left(L_{i}^{\prime}\right), E \subset \bigcup_{i=1}^{\infty} L_{i}^{\prime}, L_{i} \in \mathcal{L}\right\}$.

DEFINITION 3.5 Let $\mathcal{L}$ be a lattice of subsets of $x$, let $\mu \in I(\mathcal{L})$ and $E \subset X$ and define $\tilde{\widetilde{\mu}}(E)=\inf \left\{\sum_{i=1}^{\infty} \mu\left(L_{i}\right), E \subset \bigcup_{i=1}^{\infty} L_{i}, L_{i} \in \mathcal{L}\right\}$.

REMARK Both $\mu$ " and $\tilde{\tilde{\mu}}$ are outer measures on $\mathrm{P}(\mathrm{X})$, clearly. If $\mu \in \mathrm{I}(\boldsymbol{\mathcal { Z }})$ but $\mu \notin I_{\sigma}(\mathscr{L})$ then $\mu$ " $\exists 0$. If $\mu \in I_{\sigma}(\mathscr{L})$ then $\mu \leqslant \mu "(\mathscr{L})$. Similar remarks for $\widetilde{\widetilde{\mu}}$.

THEOREM 3.4 Let $\mathscr{L}, \subset \mathscr{L}_{\mathbf{2}}$ be two lattices of subsets of $\mathrm{x}$ such that $\mathscr{L}_{1}$ semiseparates $\mathscr{L}_{2}$. Suppose that $\mathscr{L}_{1}$ is $\delta$ and let $\mu \in \mathrm{I}_{\mathrm{R}}^{\sigma}\left(\mathscr{L}_{1}\right)$. Then $\mu^{\prime \prime}=\widetilde{\widetilde{\mu}}\left(\mathscr{L}_{2}\right)$. 
PROOF. Clearly, $\mu$ " $\leqslant \mu^{\prime}$. Let $\in \mathscr{L}, \delta$-lattice so that $L=\bigcap_{i=1}^{\infty} L_{i}, L_{i} \mathscr{L}_{1}$, and let $\mu \in I_{R}^{\sigma}\left(\mathscr{L}_{1}\right)$. Then $\mu\left(L^{\prime}\right)=\mu\left(\left(\sum_{i=1}^{\infty} L_{i}\right)^{\prime}\right)=\mu\left(\sum_{i=1}^{\infty} L_{i}^{\prime}\right) \leqslant \sum_{i=1}^{\infty} \mu\left(L_{i}^{\prime}\right)$ since $\mu$ countably additive; therefore $\mu^{\prime}=\mu^{\prime \prime}$. By Theorem 3.3 it follows $\mu^{\prime \prime}=\mu^{\prime}=\tilde{\mu}\left(\mathscr{L}_{2}\right)$. But since $\tilde{\tilde{\mu}} \leq \tilde{\mu}$ everywhere, we get $\tilde{\widetilde{\mu}} \leqslant \mu$ " $\left(\mathscr{L}_{2}\right)$. Suppose $\widetilde{\tilde{\mu}}\left(L_{2}\right)=0$ with $L_{2} \in \mathscr{L}_{2}$, but $\mu^{\prime \prime}\left(L_{2}\right)=1$. Then $L_{2} \subset \bigcup_{i=1}^{\infty} A_{i}, A_{i} \in \mathscr{L}$, and $\mu\left(A_{i}\right)=0$ all $i$. By the $\mathscr{L}$-regularity of $\mu$ we have $A_{i} \subset B_{i}^{\prime}$, $B_{i} \in \mathscr{L}_{1} \approx$ and $\mu\left(B_{i}^{\prime}\right)=0$. Therefore $L_{2} \subset \bigcup_{i=1}^{\infty} B_{i}^{\prime}, \mu\left(B_{i}^{\prime}\right)=0$ and $\mu$ " $\left(L_{2}\right)=0$, contradiction. Hence $\tilde{\tilde{\mu}}=\mu "\left(\mathscr{L}_{2}\right)$.

Further related material can be found in [4-6].

4. I-LATTICES

DEFINITION 4.1 A lattice $\mathcal{L}$ is called an 1 -lattice if every $\mathscr{L}$-filter with the countable intersection property is contained in an $\mathscr{L}$-ultrafilter with the countable intersection property (i.e. for $\pi \in \widetilde{\pi}_{G}(\mathscr{L})$ there exists $\mu \in I_{R}^{\widetilde{\sigma}}(\mathscr{L})$ such that $\pi \leqslant \mu(\mathscr{L})$ )

THEOREM 4.1 If $\mathscr{L}$ is an I-lattice and replete then $\mathscr{L}$ is Lindelof.

PROOF. Let $\pi \in \pi_{\mathscr{G}}(\mathcal{L})$. There exists $\mu \in I_{R}^{\sigma}(\mathscr{L})$ with $\pi \leqslant \mu(\mathscr{L})$. $\mathscr{L}$ replete implies $s(\mu)=\bigcap_{\alpha \in \Lambda}\left\{L_{\alpha} \in \mathscr{L} / \mu(L)=1\right\} \neq \emptyset$, therefore $S(\mu)<s(\pi) \neq \emptyset$. But $\pi \in \pi_{\xi}(\mathcal{L})$ and then $\bigcap_{\alpha=1}^{\infty}\left\{L_{\alpha} \in \mathscr{L} / \pi\left(L_{\alpha}\right)=1\right\} \neq \emptyset$ i.e. $\mathscr{L}$ is Lindelof.

THEOREM 4.2 If $\mathscr{L}$ is a countably compact lattice then $\mathscr{L}$ is an I-lattice.

PROOF: Let $\left\{L_{\alpha}\right\}_{\alpha=1}^{\infty}$ be a collection of subsets of $x$ such that $\bigcap_{\alpha=1}^{n}\left\{L_{\alpha}\right\} \neq \emptyset$. Since $\mathcal{L}$ countably compact, $\bigcap_{\alpha=1}\left\{L_{\alpha} \in \mathcal{L}\right\} \neq \emptyset$ and then $\left\{L_{\alpha}\right\}_{\alpha}$ is a filter base which generates an $\mathscr{L}$-filter with the countable intersection property, $\pi \in \mathbb{T}_{\mathscr{G}}(\mathscr{L})$. We enlarge it to $\mathcal{F}$, an $\mathscr{L}$-ultrafilter with the countable intersection property. To $\mathcal{F}$ it corresponds uniquely $\mu \in \mathrm{I}_{\mathrm{R}}^{\widetilde{\sigma}}(\mathscr{L})$ and $\pi \leq \mu(\mathscr{L})$.

THEOREM 4.3 If $\mathscr{L}$ is disjunctive and Lindelof then $\mathscr{L}$ is an I-lattice.

PROOF. Let $\pi \in \pi_{\sigma}(\mathcal{L})$ and let $\left\{L_{\alpha}\right\}_{\alpha \in \Lambda}$ be a family of subsets of $x$. Then $\bigcap_{\alpha=1}^{\infty}\left\{L_{\alpha} / \pi\left(L_{\alpha}\right)=1\right\} \neq \emptyset$ and since $\mathscr{L}$ is Lindel $\forall f, \bigcap_{\alpha \in \Lambda}\left\{L_{\alpha} / \pi\left(L_{\alpha}\right)=1\right\}=S(\pi) \neq \emptyset$. Let $x \in S(\pi)$ and consider $\mu_{x}$. Clearly, $\bar{\pi} \leqslant \mu_{x}(\mathscr{L})$ and $\mu_{x} \in I_{G}(\mathscr{L})$ and since $\mathscr{L}$ is disjunctive, $\mu \in I_{R}(\mathscr{L})$ Therefore $\mu_{x} \in I_{R}^{\sigma}(\mathscr{L})$.

DEFINITION 4.2 Let $\mathscr{L}$ be a disjunctive lattice of subsets of $x$ and let $\mu \in I_{R}(\mathscr{L})$ Define $\mu^{\prime}$ on $a\left(w_{\sigma}(\mathscr{L})\right)=w_{\sigma}(Q(\mathscr{L}))$ by $\mu^{\prime}\left(w_{\sigma}(A)\right)=\mu(A), A \in Q(\mathscr{L})$ where $w_{\sigma}(A)=\left\{\mu=I_{R}^{G}(\mathscr{L}) /\right.$ $\mu(A)=1\}$ and $w_{\sigma}(\mathscr{L})=\left\{w_{\sigma}(A) / A \in Q(\mathscr{L})\right\}$. Clearly, for $A, B \in Q(\mathscr{L})$ the properties a)-e) that we stated in section 3 are still valid. Note that $W_{\sigma}(\mathscr{L})$ is a disjunctive lattice. The following theorem follows directly from the definitions:

THEOREM 4.4 If $\mu \in \mathrm{I}_{\mathrm{R}}(\mathscr{L})$ then $\mu \in \mathrm{I}_{\mathrm{R}}^{\mathbb{G}}(\mathscr{L})$ iff $\boldsymbol{\mu}^{\prime} \in \mathrm{I}_{\mathrm{R}}^{\widetilde{\sigma}}\left(W_{\sigma}(\mathscr{L})\right.$ ). (More generally: if $\mu \in I(\mathscr{L})$ then $\mu^{\prime} \in I\left(W_{G}(\mathscr{L})\right)$ and $\mu_{R} I_{R}(\mathscr{L})$ iff $\left.\mu^{\prime} \in I_{R}\left(W_{G}(\mathscr{L})\right)\right)$.

THEOREM 4.5 If $\mathscr{L}$ is disjunctive then $\mathscr{L}$ is an I-lattice iff $\left(\mathscr{F}_{R}(\mathscr{L}), \operatorname{tW}_{\sigma}(\mathscr{L})\right)$ is Lindelof.

PROOF. Necessity: first we show that $W_{G}(\mathcal{L})$ is an I-lattice. Let $\pi \in \widetilde{K}_{\mathscr{\sigma}}(\mathscr{L})$. There exists $\mu \in I_{R}^{\sigma}(\mathscr{L})$ with $\pi \leqslant \mu(\mathscr{L})$. Hence by Theorem 4.4 we have $\pi^{\prime} \in \widetilde{\pi}_{G}\left(w_{G}(\mathscr{L})\right)$ and $\mu^{\prime} \in I_{R}^{\sigma}\left(W_{\sigma}(\mathscr{L})\right)$ with $\pi^{\prime} \leqslant \mu^{\prime}\left(W_{\sigma}(\mathscr{L})\right)$. Since $\mathscr{L}$ is disjunctive, $W_{G}(\mathscr{L})$ is replete and by Theorem 4.1 it follows that $W_{\sigma}(\mathscr{L})$ is Lindeluf. $W_{\sigma}(\mathscr{L}) \subset \operatorname{tW}_{\sigma}(\mathscr{L})$ implies that $\operatorname{tW}_{B}(\mathscr{L})$ is Lindel $\forall f$. Sufficiency: $\operatorname{tW}_{\sigma}(\mathscr{L})$ Lindel $\forall f$ implies that $W_{G}(\mathscr{L})$ Lindel $\forall f$ and since $W_{\sigma}(\mathscr{L})$ is disjunctive, by Theorem 4.3 it follows that $W_{\sigma}(\mathscr{L})$ is an I-lattice. Therefore for $\pi^{\prime} \in \pi_{\sigma}\left(W_{\sigma}(\mathscr{L})\right)$ there exists $\mu^{\prime} \in I_{R}\left(W_{\sigma}(\mathscr{L})\right)$ such that $\pi^{\prime} \leq \mu^{\prime}\left(W_{\sigma}(\mathscr{L})\right)$. To $\pi^{\prime}$ and $\mu^{\prime}$ correspond $\pi \in \mathbb{F}_{\mathcal{G}}(\mathscr{L})$ and $\mu \in \mathrm{I}_{\mathrm{R}}^{\mathcal{G}}(\mathscr{L})$ such that $\pi \leq \mu(\mathscr{L})$.

THEOREM 4.6 Let $\mathscr{L}, \subset \mathscr{L}_{2}$ be two lattices of subsets of $x$ such that $\mathscr{L}_{1}$ is $\delta$ and an I-lattice and $\mathscr{L}_{2}$ is disjunctive. Consider that the restriction $\Psi: I_{R}^{\mathscr{F}}\left(\mathscr{L}_{2}\right) \rightarrow I_{R}^{\mathfrak{F}}\left(\mathscr{L}_{1}\right)$ is closed with respect to wallman topologies. Then $\mathscr{L}_{1}$ semiseparates $\mathscr{L}_{\mathbf{2}}$. 


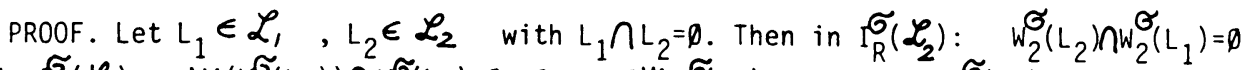

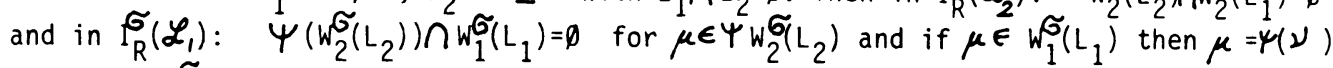
where $\nu \in W_{2}^{\mathscr{G}}\left(\mathrm{L}_{2}\right)$ and $\mu\left(\mathrm{L}_{1}\right)=1$. But then $\nu\left(\mathrm{L}_{2}\right)=1$ and $\nu\left(\mathrm{L}_{1}\right)=1$, contradiction since $\mathrm{L}_{1} \cap \mathrm{L}_{2}=\emptyset$. Now since $\psi$ is closed $\psi W_{2} \sigma_{2}\left(L_{2}\right)=\cap_{1} \sigma_{1}\left(L_{1 \alpha}\right), L_{2} \subset L_{1 \alpha} \in \mathscr{L}_{1}$ therefore $w_{1}^{\sigma}\left(L_{1 \alpha}\right) \cap w_{1}^{\sigma}\left(L_{1}\right)=\emptyset$. Hence, since $\mathscr{L}_{1}$ is an I-lattice and disjunctive (because $\mathscr{L}_{2}$ is disjunctive), by Theorem $4.5 \quad I_{R}^{\sigma}\left(\mathscr{L}_{1}\right), w_{1}^{\sigma}\left(\mathscr{L}_{1}\right)$ is $L_{\infty}$ indelof. Now $w_{1}^{\widetilde{\sigma}}\left(\bigcap_{1} L_{1} \alpha_{i} w_{1}^{\sigma}\left(L_{1}\right)=\right.$ $=\bigcap_{\infty}^{\infty} w_{1}^{\sigma}\left(L_{1 \alpha_{i}} \cap w_{1}^{\sigma}\left(L_{1}\right)=\varnothing\right.$ and then $\cap L_{1 \alpha_{i}} \cap L_{1}=\emptyset$, where $\bigcap_{1}^{\infty} L_{1 \alpha_{i}} \in \mathcal{L}_{1}$ which is $\delta$ and $\overbrace{1} L_{L_{i}}{ }^{\partial} L_{2}$. Hence $\mathscr{L}_{1}$ semiseparates $\mathscr{L}_{2}$.

Here we give conditions which guarantee that $\psi$ is basically closed.

DEFINITION $4.3 \quad \mathscr{L}_{2}$ is countably bounded $\mathscr{L}_{1}$ lattice if for $A_{n} \in \mathscr{L}_{2}, n=1,2, \ldots$ and $A_{n} \downarrow \theta$, there exists $B_{n} \in \mathscr{L}_{1}, n=1,2, \ldots$ with $A_{n} \subset B_{n}$ and $B_{n} \downarrow \theta$.

THEOREM 4.7 Let $\mathscr{L}_{1} \subset \mathscr{L}_{2}$ be two lattices of subsets of $X$ such that $\mathscr{L}_{1}$ semiseparates $\mathscr{L}_{2}, \mathscr{L}_{2}$ is $\mathscr{L}_{1}$-countably bounded and $\mathscr{L}_{2}=t \mathscr{L}_{1}$. Then the restriction $\Psi: I_{R}^{\mathscr{G}}\left(\mathscr{L}_{2}\right) \rightarrow I_{R}^{\widetilde{\sigma}}\left(\mathscr{L}_{1}\right)$ is basically closed.

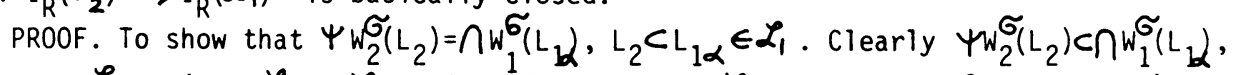
$\mathrm{L}_{2} \subset \mathrm{L}_{1 \alpha} \in \mathscr{L}_{1}$ since $\mathscr{L}_{2}=\mathrm{t} \mathscr{L}_{1}$ i.e. for any $\mathrm{L}_{2} \in \mathscr{L}_{2}$ we have $\mathrm{L}_{2}=\alpha_{\alpha} \mathrm{L}_{1 \alpha}, \mathrm{L}_{1 \alpha} \in \mathscr{L}_{1}$. Now let $\mu=\psi(\nu) \in \cap w_{1}^{\sigma}\left(L_{2}\right)$, but $\mu \notin \Psi w_{2}^{\sigma_{2}}\left(L_{2}\right)$. Therefore $\mu \in I_{R}^{\sigma}\left(\mathscr{\alpha}_{1}\right)$ and since $\mathscr{L}_{2}$ is . $\mathscr{L}_{1}$ countably bounded, $\nu \in \mathbb{F}_{R}^{\widetilde{\sigma}}\left(\mathscr{L}_{2}\right)$. So, $\mu\left(L_{1 \alpha}\right)=1$ all $L_{\alpha \alpha} L_{2}$ but $\nu\left(L_{2}\right)=0$. Since then $\nu\left(L_{2}^{\prime}\right)=1$ and $\nu \in I_{R}^{\sigma}\left(\mathscr{L}_{2}\right)$, there exists $\tilde{L}_{2} \subset L_{2}^{\prime}, \tilde{L}_{2} \in \mathscr{L}_{2}, \nu\left(\widetilde{L}_{2}\right)=1$. By semiseparation there exists $\tilde{L}_{1} \in \mathscr{L}_{1}, \tilde{L}_{2} \subset \tilde{L}_{1}$ and $L_{1 \alpha} \cap \tilde{L}_{1}^{2}=\emptyset$. But $\tilde{L}_{2} \subset \tilde{L}_{1}$ and $\nu\left(\tilde{L}_{2}\right)=1$ implies $\mu\left(\tilde{L}_{1}\right)=1$ and since also $\mu\left(\mathrm{L}_{1 \alpha}\right)=1$ all $L_{1 \alpha}$, it follows $\mu\left(\tilde{L}_{1} \cap L_{1 \alpha}\right)=1$ which contradicts that $\mathrm{L}_{1 \alpha} \cap \tilde{\mathrm{L}}_{1}=\emptyset$.

\section{REFERENCES}

1. ALEXANDROFF, A.D. Additive Set Functions in Abstract Spaces, Mat.Sb. (N.S) 8, 50 (1940), 307-348.

2. VLAD, C. Direct Product of Measures, Libertas Mathematica, Univ. of Texas, Vol. VII(1987), 71-84.

3. VLAD, C. Lattice Separation and Properties of Wallman Type Spaces, Annali di Matematica Pura ed Applicata ( to appear).

4. HUERTA, C. Measure Requirements on Distributive Lattices for Boolean Algebras and Topological Applications, Proc. Amer. Math.Soc. 106, no.2,1989,307-309.

5. EID, G.M. On Normal Lattices and Wallman Spaces, Internat.J.Math \& Math. Sci. 13(1), (1990), 31-38.

6. FROLIK, Z. Prime Filters with the C.I.P., Comm.Math.Univ. Carolinae, 13(1972). 553-575. 


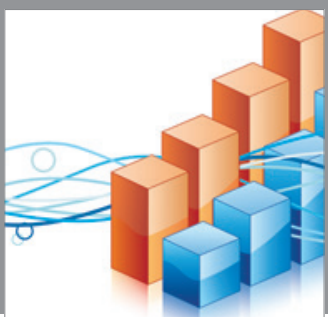

Advances in

Operations Research

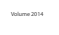

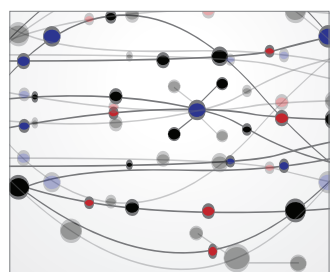

\section{The Scientific} World Journal
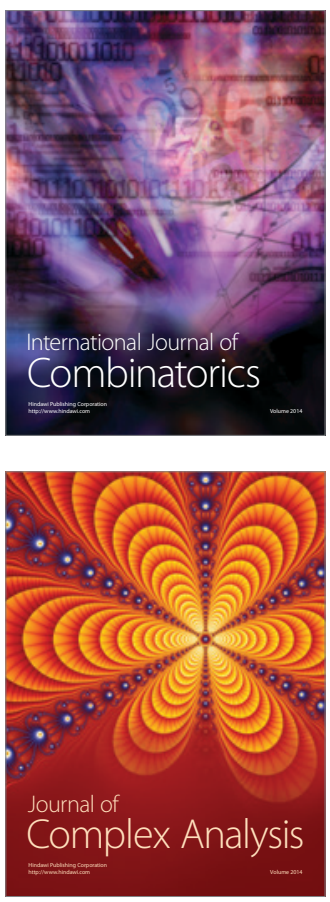

International Journal of

Mathematics and

Mathematical

Sciences
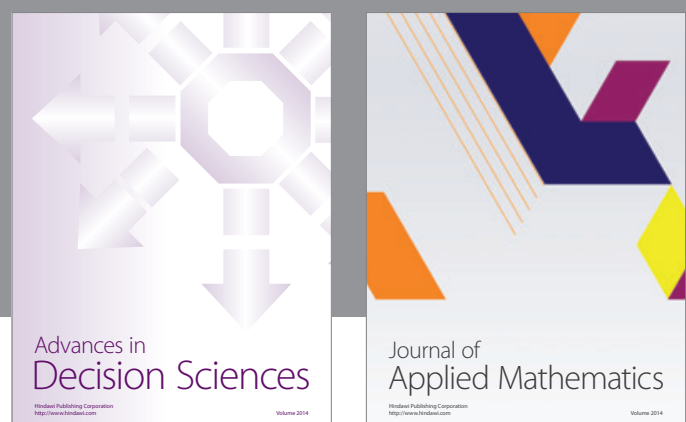

Journal of

Applied Mathematics
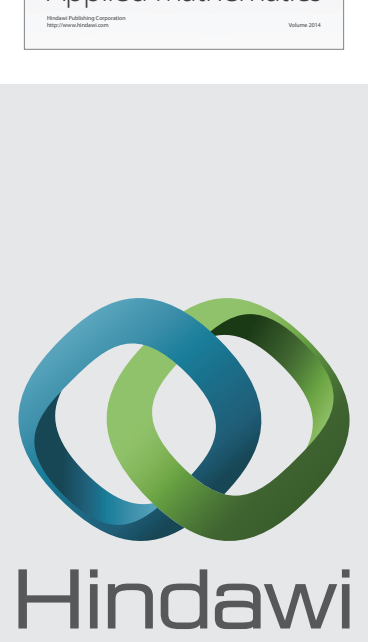

Submit your manuscripts at http://www.hindawi.com
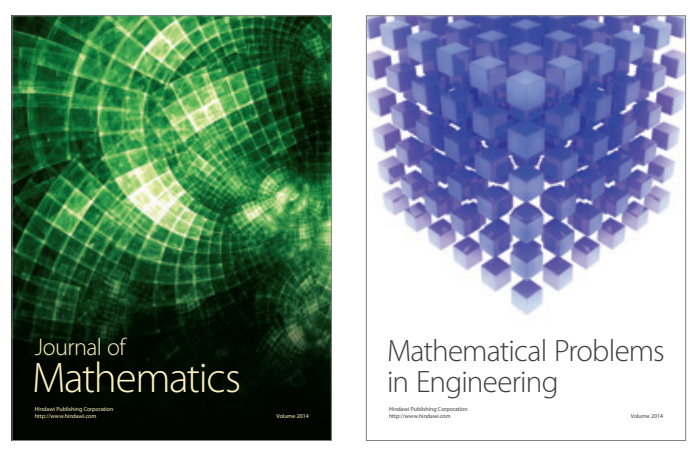

Mathematical Problems in Engineering
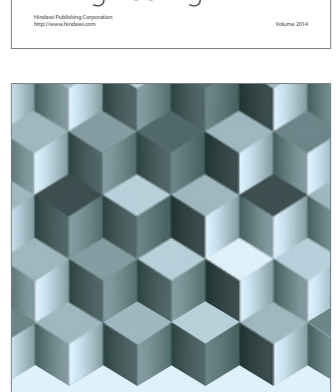

Journal of

Function Spaces
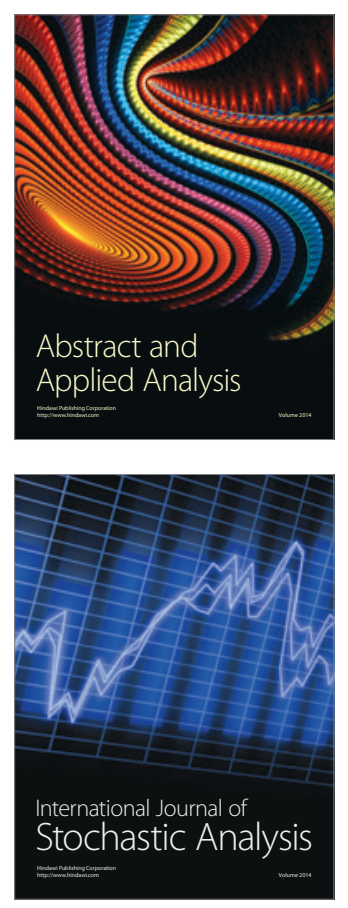

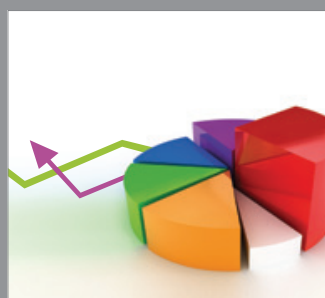

ournal of

Probability and Statistics

Promensencen
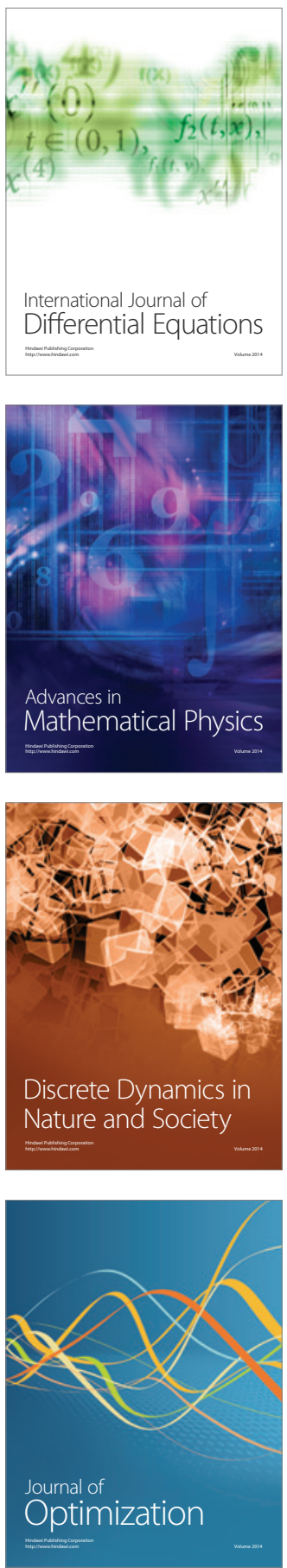